\title{
Sistema Imersivo para Pessoas com Deficiência
}

\author{
Sandra Dutra Piovesan ${ }^{1}$, Rosana Wagner ${ }^{2}$, \\ Roseclea Duarte Medina ${ }^{2}$, Liliana Maria Passerino \\ ${ }^{1}$ Curso de Graduação em Engenharia da Computação - Universidade Federal do Pampa \\ (UNIPAMPA) Bagé - RS - Brasil \\ ${ }^{2}$ Programa de Pós Graduação em Informática na Educação - Universidade Federal do \\ Rio Grande do Sul (UFRGS) Porto Alegre - RS - Brasil \\ \{sanpiovesan, rosanawagner, roseclea.medina, lpasserino\}@gmail.com
}

\begin{abstract}
This article aims to present software that uses the concepts of Virtual Reality Environment using Immersive focusing on the preparation and development of people with disabilities for inclusion in the labor market. The immersive environment or world is nearing completion of implementation and early use with a group of students who exercise their work in a company survey participant. It is believed that the virtual world will contribute to the development and interactions that these students will have in the real world, working as a system of Alternative Communication.
\end{abstract}

Resumo. Este artigo tem como objetivo apresentar o software que utiliza os conceitos de Realidade Virtual usando um ambiente imersivo com foco na preparação e desenvolvimento das pessoas com deficiência para inclusão no mercado de trabalho. $O$ ambiente imersivo ou mundo está em fase de testes e uso precoce com um grupo de estudantes que exercem o seu trabalho em uma empresa participante da pesquisa. Acredita-se que o mundo virtual vai contribuir para o desenvolvimento e as interações que esses alunos terão no mundo real, funcionando como um sistema de comunicação alternativa.

\section{Introdução}

A inclusão das pessoas com deficiência no mercado de trabalho pode ser um diferencial na qualidade de vida desses indivíduos, sendo que a participação de forma igualitária em sociedade, respeitando os direitos e obrigações ainda é um desafio.

A inclusão social ganhou força nas últimas décadas em todos os setores da sociedade e principalmente na educação (BEYER, 2005). Os avanços evidenciados nas tecnologias de informação e comunicação possibilitaram a construção de ambientes adaptados e flexíveis para atender o contexto dos estudantes (PASSERINO, 2006).

No âmbito do trabalho, os avanços começam a aparecer a partir do ano de 1991 quando se promulgou no Brasil a lei $n^{\circ} 8.213$ que determina que as empresas com mais de 100 funcionários devem contratar pessoas com deficiências. A lei, conhecida como a Lei de Cotas, prevê que uma quantidade de vagas deve ser reservada para pessoas com deficiência, que varia de $2 \%$ a $5 \%$ do número total de funcionários, mas devido a vários motivos esta lei nem sempre é seguida. 
Pesquisas mostram que estabelecimentos com menos de 100 funcionários, que por lei não têm obrigação de contratar pessoas com deficiência, apresentam uma taxa de empregabilidade média de 1,05\%. As empresas empregadoras de 100 a 200 funcionários são obrigadas a reservar $2 \%$ de seus postos para pessoas com deficiência, apresentam uma taxa de empregabilidade média de 2,7\%. Nas empresas com 201 a 500 empregados, verifica-se uma taxa de empregabilidade de $2,9 \%$ contra a cota exigida por lei de 3\%. Quando são examinadas empresas que possuem de 501 a 1000 empregados, observamos uma taxa de empregabilidade de $2,8 \%$ contra $4 \%$ da cota exigida. Descumprimento similar à lei é observado nas empresas com mais de 1000 funcionários, pois a legislação determina que essas empresas ofereçam pelo menos 5\% de seus postos de trabalho às pessoas com deficiência contra $3,6 \%$ da taxa de empregabilidade média observada na prática (SANTOS, 2013).

A importância da qualificação, neste cenário de inclusão é evidente. Teperino ressalta a dificuldade das pessoas com deficiência em ter acesso à escolaridade como um dos fatores que mais podem comprometer o seu ingresso no mercado de trabalho (IBDD, 2003)(PEREIRA, 2013).

Neste sentido que as tecnologias voltadas à qualificação das pessoas com deficiência podem ser uma importante ferramenta para o acesso das pessoas com deficiência ao mercado de trabalho. Para Grau (1995), a tecnologia é uma atividade humana que tem por finalidade inventar e projetar artefatos e processos que permitam converter recursos materiais, humanos e de informação em formas e dispositivos para solucionar as necessidades sociais e dos seres humanos individualmente (PEREIRA, 2013).

As inovações tecnológicas podem alterar o modo de vidas das pessoas, interferindo assim no sistema social e na cultura, tendo um papel fundamental no desenvolvimento humano, perpassando pelo seu uso para a promoção da inclusão social (PASSERINO, 2010). Dessa forma, a tecnologia pode ser entendida como recurso amplo de acesso a todas as instâncias sociais, com propósito da inclusão, objetivando a autonomia do indivíduo (PEREIRA, 2011).

A área da tecnologia que se destina especificamente à ampliação de habilidades de comunicação é denominada de Comunicação Alternativa (CA). A comunicação alternativa destina-se a pessoas sem fala ou sem escrita funcional ou em defasagem entre sua necessidade comunicativa e sua habilidade de falar e/ou escrever (BERSCH, 2013).

Nas ultimas décadas surgiram experiências pedagógicas realizadas em mundos virtuais, mais especificamente em mundos virtuais $3 \mathrm{D}$ on-line, que chamam a nossa atenção pelo grau de envolvimento e imersão dos estudantes com os conteúdos dos cursos, com os colegas e com o próprio professor, que não é reproduzido nos tradicionais AVAs: Moodle, Teleduc, Amadeus, etc. (MATTAR, 2008). Em um ambiente virtual $3 \mathrm{D}$, você vê o seu próprio avatar. Na informática, avatar é um cibercorpo inteiramente digital, uma figura gráfica de complexidade variada que empresta sua vida simulada para o transporte de cibernautas para dentro dos mundos paralelos do ciberespaço (SANTAELLA, 2003). Você está situado, vê e ouve coisas daquele ponto de vista e então parece estar presente naquele ambiente (MATTAR, 2008). 
O uso deste tipo de ambiente no âmbito laboral é comum especialmente para treinamentos específicos e de alto risco como simuladores de vôo, treinamento militar, na medicina e na aviação, em campos onde decisões e ações dos aprendizes podem causar danos ou mortes (MARINS, 2007). Porém, poucos são os estudos do uso de ambientes de RV para a inclusão das pessoas com deficiência no mercado de trabalho. Diante disso, se tem por objetivo o desenvolvimento de um ambiente que se utiliza dos conceitos de Realidade Virtual e Ambientes Imersivos para a qualificação de pessoas com deficiência visando compreender os processos de aprendizagem e desenvolvimento desses indivíduos na preparação para o trabalho quando mediados por ambientes de RV.

\section{Realidade Virtual: Conceitos Básicos}

A evolução constante da tecnologia está levando a educação para novos caminhos, bem mais atrativos aos estudantes, tornando possível a utilização de novas ferramentas, levando a uma evolução no processo de ensino e aprendizagem. A Realidade Virtual ocupa um importante lugar nessa evolução.

A Realidade virtual e suas variações representam técnicas de interface computacional que levam em conta o espaço tridimensional. Nesse espaço, o usuário atua de forma multissensorial, explorando aspectos deste espaço por meio da visão, audição e tato. Conforme a tecnologia disponível é possível também explorar o olfato e o paladar. Percepções corpóreas, como frio, calor e pressão, estão incluídas no tato, através da pele (KIRNER, 2011)(KIRNER, 2012).

Na Realidade Virtual por imersão, o utilizador tem a sensação real de estar dentro do mundo virtual e que é capaz de manipular os objetos ali presentes como se eles fossem reais, visto que com o desenvolvimento tecnológico, estes objetos passaram a responder às interações realizadas pelo usuário (BRAGA, 2001).

Ambientes imersivos são os espaços que utilizam sistemas computacionais para promover um estado de ilusão perceptiva e que incitam o visitante à participação. A imersão é usada como um estágio para aniquilar a diferença entre realidade e representação e como instrumento de persuasão da mente fazendo a passagem entre o realismo e o fantasioso (MEDEIROS, 2005).

Outros benefícios são observados com o uso de Realidade Virtual na educação. Segundo Clark (2006) a Realidade Virtual pode ser usada para tornar o aprendizado mais interessante e divertido com o objetivo de melhorar a motivação e a atenção, reduzindo custos quando a utilização do objeto e do ambiente real for mais dispendiosa que a simulação. Também possibilita que se explorem situações que são impossíveis de serem feitas no mundo real, por exemplo: explorar um planeta como Marte, viajar dentro do corpo humano, fazer explorações submarinas ou dentro de cavernas, visitar lugares muito pequenos para serem vistos (moléculas) ou muito caros ou muito distantes, ou ainda porque esse lugar está no passado (lugares históricos). Outro fator de destaque na RV é a melhoria de transferência de conhecimento, onde há a possibilidade de acessar o conteúdo de aprendizagem em qualquer lugar e em qualquer tempo (em caso de uso de Realidade Virtual na Internet), eliminando em casos de experimentos os riscos e perigos para o ambiente, para o professor ou para o aprendiz. Devido a esse último benefício, redução de riscos de acidentes, as simulações têm sido cada vez mais 
utilizadas em treinamento militar, na medicina e na aviação, em campos onde decisões e ações dos aprendizes podem causar danos ou mortes (MARINS, 2007).

\subsection{Laboratório de Modelagem}

OpenSim faz parte do Projeto OpenSimulator (OS), é um servidor de Mundos Virtuais que pode ser utilizado para criar e desenvolver Ambientes Virtuais em 3D. Ele vem sendo desenvolvido por vários programadores. Pronto para uso, o OpenSimulator pode ser utilizado para criar um ambiente semelhante ao Second Life. Ele também pode ser facilmente estendido para produzir aplicações interativas em 3D mais especializadas. A interação acontece através de bate-papos e observação das ações de outros Avatares (http://opensimulator.org).

OpenSim é uma solução de código aberto, o que oferece alta compatibilidade com Second Life e com protocolos de comunicação de outras plataformas. É amplamente utilizado por universidades e grandes empresas (por exemplo, IBM, Microsoft). Sendo uma fonte aberta, o aplicativo concede aos designers a liberdade de criação de uma multiplicidade de interfaces de usuário, simulações e ambientes. Além disso, é especialmente adequado para a pesquisa, porque através da criação de um servidor privado, podemos ter experiências sem influência externa de outros. Em adicionalmente, o servidor de SL oficial restringe o número de objetos e o tamanho de scripts, dependendo do tamanho da área de terras de propriedade. Em OpenSim não existem tais restrições (MEDEIROS, 2005).

As Figuras 1 e 2 apresentam um ambiente imersivo desenvolvido utilizando o Simulador OpenSim. A Figura 1 apresenta a entrada no ambiente, proporcionando ao estudante a sensação real de estar no ambiente.

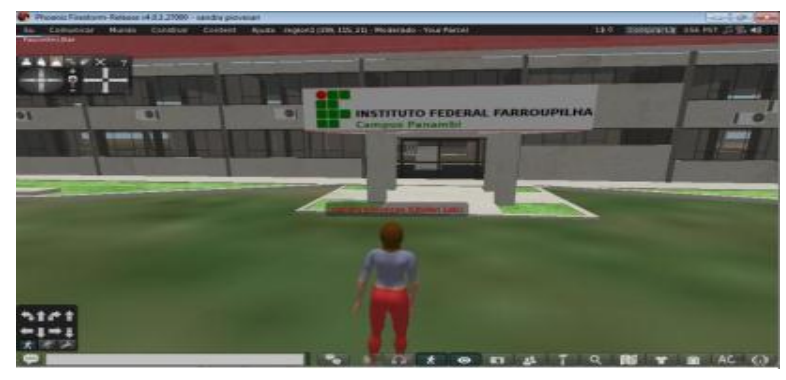

Figura 1. Entrada no Ambiente Imersivo

A Figura 2 apresenta a sala de estudos, dentro do prédio modelado, onde os estudantes tem a opção de interagirem com os equipamentos e com os outros estudantes virtualmente.

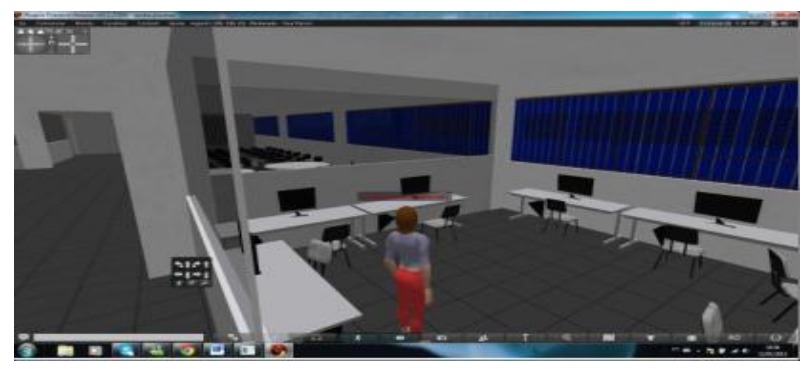

Figura 2. Sala de Estudos 


\section{A Realidade Virtual: pessoas com deficiência no trabalho}

Entre os projetos encontrados que se utilizam de RV para desenvolver habilidades sociais e cognitivas está o AViSS (Ambiente Virtual de Ensino Social e Habilidades) (EHRLICH, 2009), da Universidade do Kansas. Neste projeto foi desenvolvido um ambiente para autistas e asperges para possibilitar o desenvolvimento social, levando em consideração que esses sujeitos são visuais. Foi utilizado o simulador e visualizador OGRE e funciona tanto em Linux, Windows ou Mac. Nele os sujeitos são expostos a situações e espera-se a reação para o sistema responder ao sujeito. Também é possível rever as cenas onde as interações ocorreram. A Figura 3 apresenta uma tela do sistema AViSS.

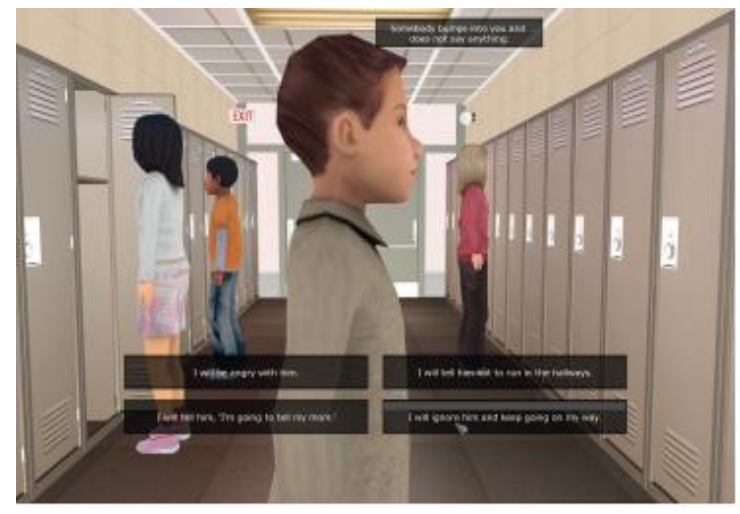

Figura. 3. AViSS (EHRLICH, 2009).

Outro projeto encontrado que mostra os benefícios da utilização da RV para o desenvolvimento de autistas é o projeto desenvolvido por Grynszpan (GRYNSZPAN, 2011). Neste projeto foi utilizado eyetracking (óculos para RV) e como resultado o projeto demonstrou que autistas tendem a visualizar a área da boca quando estão em uma conversa, enquanto outras pessoas tem o foco no olhar. Os testes foram realizados tanto com adolescentes como crianças onde eram apresentados diálogos escritos juntamente com expressões faciais onde os sujeitos deveriam clicar nas alternativas corretas. A Figura 4 apresenta uma tela do sistema desenvolvido.

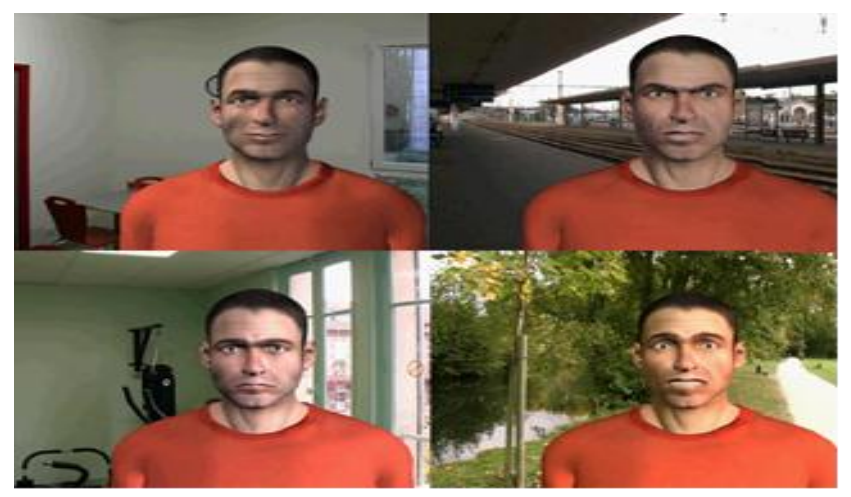

Figura. 4. Sistema de Expressões Faciais (GRYNSZPAN, 2011).

Também foram encontrados trabalhos que buscam identificar e analisar quais são os benefícios da utilização dos ambientes tridimensionais, como é o caso da pesquisa de Andreas el. al. em 2010, que busca resultados na utilização do AVA tridimensional SLoodle para a aprendizagem colaborativa, e de Guomin e Jianxin 
também em 2010, que realizou uma pesquisa com objetivo de analisar o valor educativo do ambiente SLoodle, mostrando as vantagens e os desafios encontrados.

Dentre todos os trabalhos encontrados que englobam RV e pessoas com deficiência, nenhum deles apresenta uma pesquisa relacionada ao ambiente laboral, proporcionando o ineditismo desta pesquisa.

\section{Desenvolvimento do Sistema Imersivo}

A pesquisa está sendo realizada dentro do Grupo Teias - UFRGS, e é uma Pesquisa Qualitativa e de Desenvolvimento Tecnológico.

Numa primeira etapa foi realizado um estudo de diferentes ambientes imersivos a fim de identificar o mais adequado, assim como estudos teóricos e de estado da arte sobre o uso deste tipo de ambiente com pessoas com deficiência. Também foi realizado um laboratório de modelagem, utilizando o Instituto Federal Farroupilha como teste. Durante a modelagem procurou-se realizar a mesma da forma mais fiel possível, trazendo para o mundo virtual, um ambiente fiel, muito próximo do ambiente real.

Apesar dos testes feio, o servidor de mundos virtuais OpenSim apresentou alguns problemas na importação dos modelos criados, trazendo dificuldades para manter as dimensões corretas de cada objeto dentro do mundo. A partir disso procuraram-se outras opções para importação da modelagem da empresa selecionada. Trabalhou-se então com um motor de jogo, o UDK.

Segundo Becker (2011, p.29) as finalidades como o processamento da inteligência artificial, o gerenciamento de som e música, a renderização de objetos na tela e a simulação física em jogos, podem ser considerados motores uma vez que realizam tarefas especialistas que podem ser utilizas para diversos fins (BECKER, 2011).

No que tange aos motores de jogos comerciais com versões gratuitas é possível destacar o Unreal Development Kit ou UDK4. A UDK é uma derivação da Unreal Game Engine, criada pela empresa Epic, e trata-se de uma versão gratuita do motor que deu origem aos jogos Unreal Tournament, Gears of War e mais recentemente ao jogo Dishonored. Uma característica interessante e que deve ser salientada como ponto forte é que a UDK é uma suíte completa para o desenvolvimento de jogos. Ela possui um editor visual completo que permite ao desenvolvedor concentrar todo o processo produtivo em uma única ferramenta. Além disso, a UDK introduz um sistema de programação baseado em um script próprio chamado Unreal Script. Dessa maneira, boa parte da lógica da aplicação a ser desenvolvida, e que em uma eventualidade não possa ser coberta pelo editor, pode ser facilmente programada por qualquer desenvolvedor com conhecimentos em lógica de programação, uma vez que o Unreal Script permite delegar funcionalidades sem que haja a necessidade do uso extensivo da linguagem de programação nativa que deu origem ao motor. Em tempo, os termos de uso da ferramenta se tornam gratuitos para fins didáticos e para o desenvolvimento de aplicações sem fins lucrativos (BENIN, 2012). A partir da seleção do motor UDK, foi concluída a modelagem da empresa selecionada.

$\mathrm{Na}$ busca por parceiros para a pesquisa, foi selecionada uma empresa que está interessada em participar do projeto, disponibilizando a sua estrutura para ser modelada 
e onde estão sendo realizadas as intervenções com os sujeitos que participam da pesquisa.

Após a definição da área de serviço e do campo de trabalho foi desenvolvido o ambiente em definitivo, reproduzindo da forma mais fiel possível o ambiente de trabalho escolhido. Nesta definição a semelhança é de ambiente, dos objetos e até dos avatares que participam das interações, reproduzindo assim o ambiente que o sujeito encontrará futuramente no ambiente de trabalho.

Para a modelagem foi feito um acesso ao local, à empresa, para filmagens e fotografias, assim como um acompanhamento por um curto período o trabalho em si de forma a modelar todos os elementos necessários à rotina de trabalho esperada.

Após a modelagem completa foram realizadas 3 avaliações do ambiente pelos próprios funcionários da empresa selecionada, buscando chegar ao mais próximo do real ambiente que será encontrado pelos sujeitos que participaram das pesquisas. A produção dos materiais que são utilizados dentro do "mundo" para as interações com sujeitos foi cuidadosamente modelado para que a simulação seja adequada. O sistema utiliza também vídeos com as principais funções que são desempenhadas pelos sujeitos inseridos no ambiente real de trabalho. Esses vídeos tratam das situações prováveis de acontecerem dentro da empresa, de situações pouco prováveis e também das improváveis, tentando englobar ao máximo as situações que podem ocorrer neste ambiente de trabalho.

A figura 6 apresenta a entrada do ambiente imersivo desenvolvido. Esta parte do ambiente modelado é bastante importante, pois reproduz de forma fiel o trajeto que os usuários do sistema farão quando estiverem inseridos no quadro de funcionários da empresa.

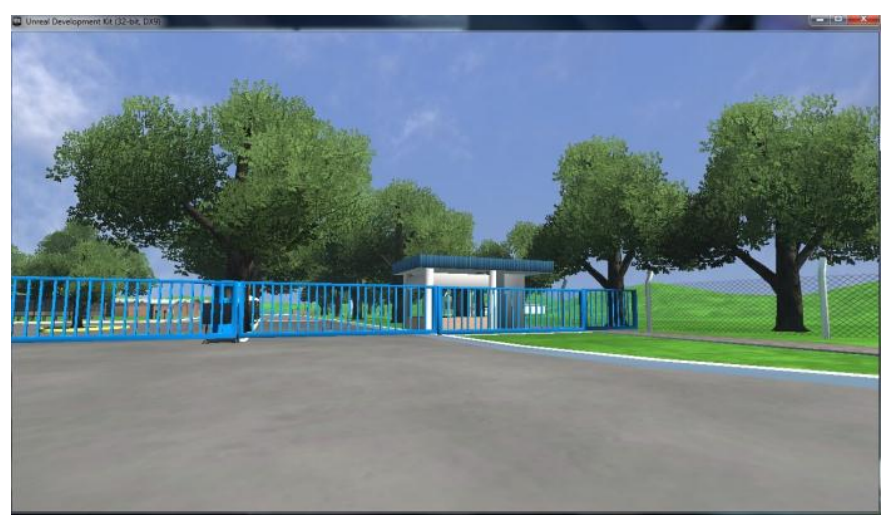

Figura. 6. Entrada do Ambiente Imersivo.

A figura 7 apresenta a estação de ônibus da empresa. A partir daqui os usuários utilizarão as catracas, que podem ser visualizadas, para a sua identificação e acesso à empresa. 


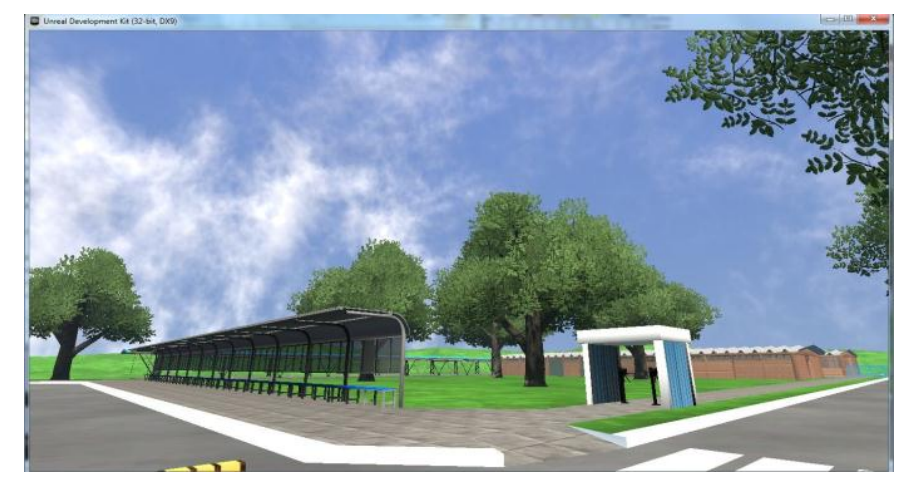

Figura. 7. Estação de ônibus.

As catracas de acesso são apresentadas na figura 8. Essas catracas estão disponíveis para os usuários que acessam a empresa pela lateral, onde há um estacionamento privativo.

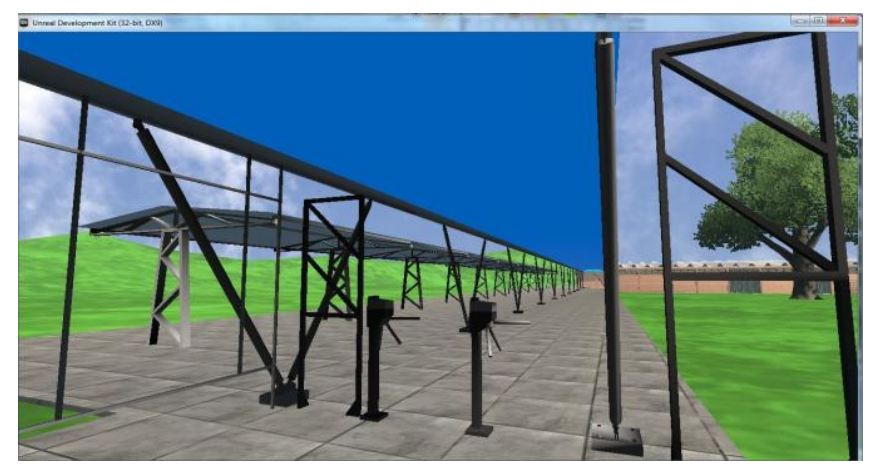

Figura. 8. Catracas laterais.

Na figura 9 é apresentado o pátio da empresa, os prédios e alguns outros detalhes como arvores, faixas de segura para pedestres e postes de luz, demonstrando a fidelidade da modelagem do ambiente.

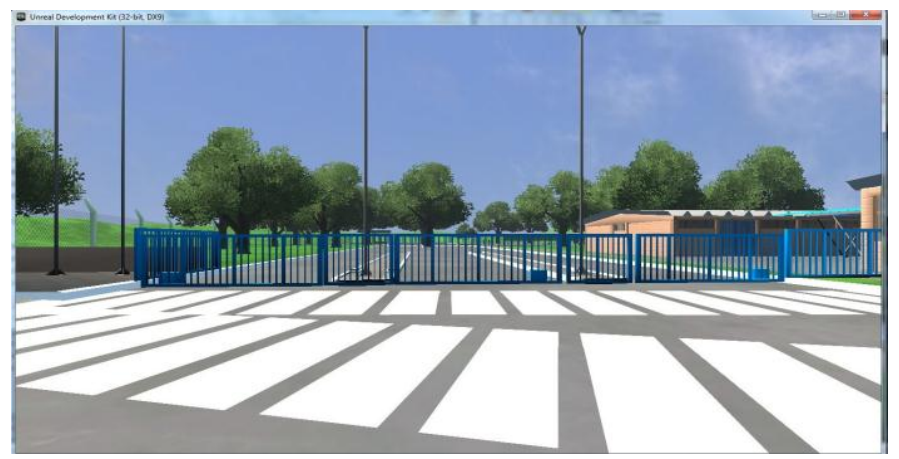

Figura. 9. Pátio da empresa.

O sistema encontra-se em fase de testes, sendo utilizado por usuários com deficiência que serão contratados pela empresa modelada. Muitas observações já foram realizadas, entre elas identificou-se um entusiasmo muito grande dos usuários pelo sistema. As interações são sempre muito prazerosas, possibilitando aos usuários uma apropriação das rotinas da empresa de forma facilitada. 


\section{Considerações Finais}

A realidade virtual, com o conceito de ambientes imersivos, já é utilizada há tempos como uma importante ferramenta com foco educacional nas mais diversas áreas de estudo. Alguns trabalhos também já demostram que pessoas com algum tipo de deficiência também podem se beneficiar do uso desses sistemas como sistemas de comunicação alternativa, facilitando a comunicação, a integração social e o desenvolvimento cognitivo dessas pessoas.

Acredita-se que o sistema desenvolvido facilitará a inserção de pessoas com deficiência no mercado de trabalho, pois simulará o ambiente real onde estes sujeitos exercerão suas atividades laborais, possibilitando o desenvolvimento de habilidades de forma prazerosa e ativa, possibilitando a apropriação de conceitos que de forma convencional seriam mais difíceis de serem aprendidos.

\section{Referências}

ANDREAS, K.; THRASYVOULOS, T.; STAVROS, D.; ANDREAS, P.; Collaborative Learning in OpenSim by Utilizing SLoodle. In: 2010 Sixth Advanced International Conference on Telecommnications, IEEE, 2010.

BEYER, H.; Inclusão e Avaliação na escola: de alunos com necessidades especiais. Porto Alegre: Mediação, 2005.

BERSCH, R.; SARTORETTO, M.,L. Assitiva: Tecnologia e Educação. Disponível online em < http://www.assistiva.com.br/ca.html >. Acesso em 18 de abril de 2013.

BECKER, H. O. Desenvolvimento de Motores de Jogos 2D: Um Estudo de Caso. Monografia. Faculdades Barddal. Florianópolis, 2011.

BENIN, M., R.; ZAMBIASI, S., P. Proposta de uma Ferramenta Focada no Ensino do Desenvolvimentode Jogos Eletrônicos. Universidade de Santa Catarina, 2012.

BRAGA, MARILUCI. Realidade Virtual e Educação. Revista de Biologia e Ciências da Terra, 2001. Acesso em

http://eduep.uepb.edu.br/rbct/sumarios/pdf/realidadevirtual.pdf, 2011.

CHANG LIU, EN YE, TIAO CHANG, YANHUI FANG. "Learning environmental science through game development in Second Life". The 2007 Ohio Digital Commons for Education (ODCE) Conference. March 5-6,2007.Columbus. Ohio. USA.

CLARK, DONALD. 2006 Motivation in e-learning. Disponível em: $<$ http://www.epic.co.uk> Março, 2012.

EHRLICH, J., A.; MILLER, J., R..A Virtual Environment for Teaching Social Skills: AViSSS. University of Kansas: IEEE Computer Society, 2009.

GRAU, J. Tecnologia y Educación. Series Aportes. Bs. As.: Fundec, 1995.

GRYNSZPAN, O., et al. A new virtual environment paradigm for high functioning autism intended to help attentional disengagement in a social contexto. França: IEEE, 2011. 
GUOMIN, Z.; JIANXIN, Z.; Na Education Value Analysis of SLoodle-based Distribuidet Virtual Learning System. In: Second International Workshop on Education Technology and Computer Science, IEEE, 2010.

IBDD (Coord.); Sem limite: inclusão de portadores de deficiência no mercado de trabalho. Rio de Janeiro: Editora Senac Rio, 2003.

KIRNER, C; Realidade Virtual e Aumentada. Acesso em Março 2012, Disponível em $<$ http://www.realidadevirtual.com.br $>$.

KIRNER, C.; KIRNER, T., G.; 2011; Evolução e Tendências da Realidade Virtual e Aumentada. In: Pré-Simposio SRV, 2011.

MARINS,V.; HAGUENAUER, C., CUNHA, G.; 2007; Realidade Virtual em educação, criando Objetos de Aprendizagem com VRML. In: Revista Digita da CVA. Volume 4, Número 5. ISSN 1519-8529.

MATTAR, J.; O Uso do Second Life como ambiente de Aprendizagem. Revista Fonte, 2008.

MEDEIROS, MARIA BEATRIZ; AMADEU, FLÁVIA. Immersive and Participative Environments. ReVISta. Arte e Conhecimento. Ano 4, n. 4 (set 2005). Brasília: IdA, 2005, p-159-171; ISSN 1518-5494.

PASSERINO, LILIANA M. Apontamentos para uma reflexão sobre a fnção social das tecnologias no processo educativo. Revista Texto Digital, Florianópolis, 2010.

PASSERINO, L., M; AVILA, B., G.; BEZ, M., R.; Scala: um Sistema de Comunicação Alternativa para o Letramento de Pessoas com Autismo. Cinted-UFRGS, 2010.

PASSERINO, L., M.; SANTAROSA, L., M., C.; Possibilidades da Mediação Tecnológica na Inclusão Escolar de Autistas. Anais do XXVI Congresso da SBC, 2006.

PEREIRA, A., C., C.. Inclusão de pessoas com deficiência no mercado de trabalho e o movimento da cultura organizacional: análise multifacetada de uma organização. Porto Alegre: UFRGS, 2011. Dissertação (Mestrado em Educação).

PAUL DOHERTY, ROB ROTHFARB,DAVE BARKER,BUILDING AN INTERACTIVE SCIENCE MUSEUM IN SECOND LIFE, in the proceedings of the Second Life Community Convention, San Francisco, USA, August 20th, 2006, pp. 19-24.

SANTAELLA, LUCIA. Cultura e artes do pós-humano, São Paulo, Paulus, 2003.

SANTOS, D. Empresas não cumprem as leis de cotas. Acesso em fevereiro de 2013. Disponível em: $\quad<$ http://www.sinthoresp.com.br/site/releases/empresas-naocumprem-a-lei-de-cotas $>$. 